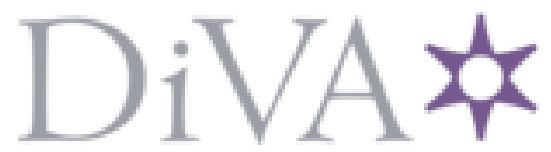

http://www.diva-portal.org

This is the published version of a paper published in International Journal of Science and Mathematics Education.

Citation for the original published paper (version of record):

Olsson, J. (2017)

The Contribution of Reasoning to the Utilization of Feedback from Software When Solving Mathematical Problems.

International Journal of Science and Mathematics Education https://doi.org/10.1007/s10763-016-9795-x

Access to the published version may require subscription.

N.B. When citing this work, cite the original published paper.

Permanent link to this version:

http://urn.kb.se/resolve?urn=urn:nbn:se:du-23800 


\title{
The Contribution of Reasoning to the Utilization of Feedback from Software When Solving Mathematical Problems
}

\author{
Jan Olsson $^{1,2}$ (D)
}

Received: 20 March 2016 / Accepted: 30 December 2016

(C) The Author(s) 2017. This article is published with open access at Springerlink.com

\begin{abstract}
This study investigates how students' reasoning contributes to their utilization of computer-generated feedback. Sixteen 16-year-old students solved a linear function task designed to present a challenge to them using dynamic software, GeoGebra, for assistance. The data were analysed with respect both to character of reasoning and to the use of feedback generated through activities in GeoGebra. The results showed that students who successfully solved the task were engaged in creative reasoning and used feedback extensively.
\end{abstract}

Keywords Dynamic software $\cdot$ GeoGebra $\cdot$ Feedback $\cdot$ Linear functions $\cdot$ Mathematical reasoning

\section{Introduction}

Even though dynamic software has been available for mathematics education for more than a decade, its potential to assist in mathematical problem-solving, compared to, for example, pen and paper and calculators, is not yet clear. One proposal for why dynamic software, for example GeoGebra, alters the conditions for learning activities like problem-solving is that it offers students the chance to interact with software. Students may, during this interaction with software, develop mathematical objects step by step, where every step is guided by the result of the previous step (Villarreal \& Borba, 2010). For example, students may construct and submit mathematical objects such as algebraic expressions into the software and receive feedback as the software draws the

Jan Olsson

jan.olsson@umu.se

1 Department of Applied Educational Science, Umeå University, Umeå, Sweden

2 Mathematics Education Research Center, Umeå University, Johan Bures väg, 90187 Umeå,

Sweden 
corresponding graph. Here, students are creating individual references for the task at hand (Mariotti, 2000). Furthermore, interacting with dynamic software to explore the problem at hand may encourage students to engage in reasoning while constructing mathematical objects to submit into the software as well as while they are interpreting the provided feedback.

However, the presence of dynamic software alone will not guarantee that students will manage to solve tasks when they do not know the path towards a solution. Dynamic software such as GeoGebra only processes what is submitted and provides feedback, the meaning of which must be interpreted. Some students may not engage in reasoning that will advance the problem-solving or utilize the appropriate feedback from GeoGebra. Insights into how different engagement in reasoning relates to successful or unsuccessful utilization of feedback from software could be important in the development of learning situations, including dynamic software. Therefore, this study investigates how students' reasoning contributes to their utilization of computergenerated feedback. Furthermore, the way in which students' reasoning and their utilization of feedback relate to success in solving mathematical tasks will be examined.

The research questions guiding this study are the following:

- How does students' reasoning contribute to their use of the feedback that GeoGebra generates?

- How do students' paths of reasoning and utilization of feedback from GeoGebra relate to their success in problem-solving?

To examine the students' reasoning and their utilization of feedback generated by GeoGebra, a didactical design (which will be presented in detail later) used in a previous study (Granberg \& Olsson, 2015) was adopted. It was designed in line with the didactical propositions of Brousseau (1997) and Schoenfeld (1985), and was shown to provide students with feedback and to invite them to engage in reasoning.

\section{Research Framework}

The theoretical concepts that will be used are presented in the following section, starting with Schoenfeld's (1985) framework for protocol analysis that will be used to structure the data. This is followed by a presentation of the theoretical concepts of imitative and creative reasoning (Lithner, 2008) and the concepts of verificative and elaborative use of feedback (Shute, 2008). These concepts will be used to analyse the data.

\section{Protocol Analysis of Problem-Solving}

Schoenfeld (1985) elaborated and extended Pólya's (1954) four problem-solving phases into the following six: reading the task, analysing (why properties of a task have certain consequences), exploration (why some outcomes will be useful), planning (why a certain approach would lead to a solution), implementing (why the problemsolving is proceeding properly) and verification (why a solution is actually reached). Furthermore, Schoenfeld (1985) proposed a method of protocol analysis to examine how a problem-solver's decisions shape the path through these phases. Schoenfeld 
divided the problem-solving process into episodes, which are periods of time during which the problem-solver is engaged in any of these phases, i.e. reading, analysing, exploring, planning, implementing or verifying. Thereafter, the transitions between these phases are identified. A transition is initiated by any of these three decision points: the junction between episodes, when new information or the opportunity to adopt a new approach appears, or when difficulties indicate that a change in approach is needed. These decisions will shape the path through these phases, i.e. through the tasksolving process. During these problem-solving phases, together with the transitions, students may be engaged in creative and imitative reasoning (Lithner, 2008).

\section{Reasoning}

The student's reasoning is defined as being her line of thought, the thinking process during which the learner successfully or unsuccessfully attempts to solve the task. Reasoning is guided and limited by the student's competences and is created in a sociocultural milieu. A student's reasoning is characterized as being imitative or creative (Lithner, 2008).

Imitative Reasoning. Reasoning is considered imitative if it consists of the use of provided or memorized facts, algorithms or procedures for how to solve the problem (Lithner, 2008). Imitative reasoning (IR) in the form of memorized reasoning (MR) is described as recalling rote-learned facts: for example, proof, a definition or a fact such as $1 \mathrm{~L}=1000 \mathrm{~cm}^{3}$. IR such as algorithmic reasoning (AR) concerns the application of provided or memorized algorithms to solve a problem. Algorithmic reasoning is often efficient to reach a correct answer, given that the algorithm is correctly implemented.

Creative Reasoning. Creative mathematical reasoning (CMR) is characterized by novelty, plausible argumentation and mathematical foundation. That is, instead of recalling a procedure that will solve the task, the students create solution methods that, at least to some extent, are new to them. The solution strategies are supported by plausible argumentation, which is anchored in intrinsic mathematical properties (Lithner, 2008). In other words, if the student, instead of applying a memorized procedure, creates an original solution method (provided it is not done through pure guesswork), it would be necessary for her to construct arguments, anchored in mathematics, for why the method may solve the task. Anchoring refers to the argument's grounding in relevant mathematical properties of the mathematical objects, transformations or concepts that the reasoning concerns. A mathematical property may be superficial or intrinsic. Lithner (2008) illustrates this point in the following example: "In deciding if $9 / 15$ or $2 / 3$ is larger, the size of the numbers $(9,15,2$ and 3$)$ is a surface property that is insufficient to consider while the quotient captures the intrinsic property" (Lithner, 2008, p. 261). Furthermore, argumentation may be considered predictive - that is to say, a mathematically anchored justification for why the strategy will work - or verificative - that is to say, a mathematically based explanation for why the solution worked or did not work. Predictive arguments will largely be observed in Schoenfeld's problem-solving phases: analysing, exploring and planning. Verificative argumentation, conversely, will primarily be observed in the phases of implementation and verification (Lithner, 2008). 
In the present study, students' reasoning during their problem-solving phases will be categorized as being either imitative or creative. Students' reasoning during the implementation and verification phases will furthermore depend on their utilization of the feedback from GeoGebra, that is, on the response given by the program when the students, for example, have submitted a formula and when GeoGebra draws the corresponding graph. To examine how students utilize the feedback from GeoGebra, Shute's (2008) concepts of verification and elaboration are used.

\section{Verificative and Elaborative Use of Feedback}

According to Shute (2008), information that is intended to be feedback (for example, a response to some action on the learner's part) can be delivered in different ways: for example, verification of response accuracy, an explanation of a correct answer, hints or worked examples. The provided feedback may thereafter be used in different ways: for example, for verification or elaboration. Verification is merely the confirmation of whether an assumption or hypothesis is correct or incorrect. Elaboration, on the other hand, can be implemented in different ways: for example, to address the response, discuss particular errors or worked examples, and so forth. One type of elaboration, response-specific use of feedback, is considered particularly efficient for learning. Response-specific use of feedback focuses on the question of why an answer is (is not) correct. Feedback can furthermore be given on various occasions during or after the learning process. In a review, Shute (2008) found that a specific form of feedback, feedback on task level, is particularly effective in supporting learning. Compared with general summary feedback, feedback on task level is more specific and often provides the student with real-time information about a particular response to a problem or task. In this study, elaboration on feedback will be characterized as being situations when the students discuss the feedback in terms of why the result was (was not) as predicted or whether the feedback is elaborated on in some other way. Furthermore, the students' use of feedback is considered verificative if they merely use information to determine whether or not they are right.

Feedback, however, is not necessarily given by a teacher or a peer. Brousseau (1997) argues that feedback could be viewed as being the result of the student's interaction with any learning milieu. That is, if the student's action changes the learning milieu, this very change may cause the student to reconsider her behaviour (Brousseau, 1997).

In the current study, GeoGebra is considered a learning milieu that will provide feedback at the task level to the student. More specifically, GeoGebra will generate information by, for example, drawing graphs according to the student's submitted formulas.

Feedback from dynamic software like GeoGebra differs from feedback provided by, for example, a teacher. Teacher feedback can generally be described as being information that is explicitly about a certain action - for example, when a teacher considers a student's attempt to solve a task and formulates feedback for the purpose of helping the student to proceed. Feedback from GeoGebra could rather be described as being implicit, an automatically generated response on students' actions (formulating and submitting input) with no explicit purpose of providing information on how to proceed. That is, the GeoGebra generated feedback is, from a student perspective, the expected or unexpected result of an activity that needs to be interpreted and that could be utilized both for verification and for elaboration. 
In this study, it is assumed that the students' reason for interacting with the software is to gain information that might help them to solve the task and that they may have a more or less articulated purpose for finding out something in particular. It is also assumed that the students will use the feedback in various ways: for example, to discover whether they are right or wrong or to find clues for how to proceed.

\section{Background}

Originally, the components of the framework used in this study (Lithner, 2008; Schoenfeld, 1985; Shute, 2008) do not explicitly consider the use of ICT. However, systematic use of digital technology in mathematics education may contribute to particular paths through problem-solving, reasoning and using feedback (Sacristán, Calder, Rojano, Santos-Trigo, Friedlander, Meissner \& Perrusquia, 2010). The use of interactive software is based on the user's existing knowledge, which will influence the medium, and the medium will influence the user. Therefore, it is important to allow students to express, present, test, refine and adjust their thinking during their tasksolving process (Hoyles, Noss, \& Kent, 2004; Lesh \& Yoon, 2004). During such activities, the learner often needs to analyse properties of mathematical objects, and a support for that is visual mathematics representations such as geometric figures, graphs and algebraic expressions (Sedig \& Sumner, 2006). The interactive contribution from dynamic software (for example, Cabri, Geometric Sketchpad, GeoGebra) is that these representations may be constructed and manipulated in direct relation to task solving.

\section{Interacting with Dynamic Software}

Interaction with dynamic software has at least two implications: the user acting upon software and software responding in some way for the user to interpret (Sedig \& Sumner, 2006). The relationship between action and response depends on whether the action is direct on an existing object or on creating an object. For example, by moving one edge of a triangle that is prepared in such a way to show the size and sum of its internal angles, the user can continuously observe the changing of the angle sizes while also observing that the sum does not change. If an object is instead created by a student submitting a command and the software in response transforms it into one or more corresponding representations, then action and response are separated and the connections between them are in some sense not transparent: that is to say, the student needs to interpret the properties of and relations between different representations. For example, if a user submits a formula for a linear function and the software transforms it into a corresponding graph, the user has to interpret the way the algebraic expression and the graph correspond. Interacting with software through commands can be described as being discrete, and the suggestion is that it is more cognitively demanding since an input object has to be formulated and submitted before the user receives any feedback (Holst, 1996). It has been found that students who explicitly express predictions for what the response could be are more successful learners (Hollebrands, 2007). Direct manipulation of objects, when the object that is manipulated changes continuously, allows users to control the flow and communication of information; however, this, on the other hand, may lead to a superficial interpretation of intrinsic properties of the 
mathematical object. In the previous example - that of manipulating a triangle (see above) - a student may accept that the sum of internal angles is $180^{\circ}$ as a fact and not reflect on properties and justifications.

\section{Dynamic Software Supporting Problem-Solving and Reasoning}

Tools like rulers, compasses and computers have always been important for students as they gain skills in mathematics. It is hard to imagine whether there would be such symbols as triangles and squares if the ruler and compass did not exist. These tools are also usable in education to reproduce such knowledge. In addition, mediating tools such as blackboards, pens and paper and textbooks are important in mathematics education. Historically, when introduced, all of them led to a slightly different perception of, and relationship to, mathematical content (Villarreal \& Borba, 2010). The introduction of dynamic software is no exception. Research has often put forward the fact that dynamic software allows students to create their own dynamic mathematical objects as references to the task at hand (Moreno-Armella, Hegedus \& Kaput, 2008). That is, instead of referring to static objects created by pen and paper or presented by teachers or textbooks, students can create and manipulate mathematical objects tailored to provide exactly the information they think they need to have in order for them to proceed with solving the task at hand (Mariotti, 2000; Moreno-Armella et al., 2008). Furthermore, the creation of mathematical objects in an environment of dynamic software such as GeoGebra and Cabri can be carried out stepwise. That means that every step is associated with an activity resulting in a response from the software, which in turn may guide the next step in task solving. Working with dynamic software means both guiding the software and being guided by the software (Moreno-Armella et al., 2008).

Several researchers advocate for how working with dynamic software promotes reasoning. Hohenwarter and Fuchs (2004) suggest that the interactive character of GeoGebra allows students to be active and solve non-routine tasks in groups or individually, and that teachers may focus on mathematical reasoning in whole-class follow-up discussions. Others emphasize students' reasoning as being connected to the work in software-for example, that tools in dynamic software can be used not only for exploration but also in support of the reasoning associated with solving the problem (Falcade, Laborde \& Mariotti, 2007). Another example is that working in dynamic software supports less strict reasoning that with the support of a teacher could be developed into deductive mathematical reasoning (Jones, 2000; Healy \& Hoyles, 2001). However, reasoning is often mentioned in general terms: that is to say, not in specific terms as to the ways in which students' reasoning contributes to activities in software (for example, in predictions and expressing purposes while formulating inputs) or as to the contributions software makes to students' reasoning (for example, in interpretations of output and justifications for solutions).

Interpreting the results of activities in dynamic software may be considered as being the use of feedback from computers. In literature, feedback from the computer is often called immediate, task associated, accurate, etc. (Sangwin, Cazes, Lee \& Wong, 2010). It is also suggested that feedback helps students to control the progress of the solution and to support explanations and justifications (Sacristán et al., 2010). Therefore, the use of feedback from dynamic software is associated with the purposes students have when it comes to creating certain activities and is related to reasoning. 


\section{Method}

The present study adopts a sociocultural perspective that considers knowledge to be skills that students develop through interactions within a social context. The method was designed to answer the research questions concerning the relation between reasoning, feedback and success in terms of solving the task. Reasoning is, in the present study, defined as being a student's train of thought: that is to say, the thinking process during which the learner successfully or unsuccessfully solves the task (Lithner, 2008). Students' reasoning can be articulated, and thereby possible to observe, as being interactions with one another, with the software, etc. Stahl (2002) proposes that these kinds of data, dialogues, computer manipulations and gestures can be merged into meaningful sequences that are possible to analyse. The reasoning of students was recorded through their conversations, computer activities and gestures and were thereafter merged into reasoning sequences that were used for analysis. The method will be presented in detail in the following section.

\section{The Didactic Situation}

The didactic situation builds on three propositions: challenge, responsibility and collaboration. Schoenfeld (1985) argues that students must work with mathematical problems that to some extent are new to them so that they can develop problem-solving skills; further, the task must constitute an intellectual challenge to the students. Brousseau (1997) proposes that if a task is to remain a challenge, students must be responsible for creating solutions of their own, and the teacher should not interfere by guiding the students towards the right answers. Brousseau furthermore notes that if a task has an appropriate design, the students will attain the target knowledge by solving the task, and they will do so only if the teacher does not provide them with the solution.

Moreover, working in small groups has been reported to be beneficial for learning when the task focuses on relationships and concepts rather than procedures. The former promotes collaboration and the latter cooperation (Lou, Abrami \& d'Apollonia, 2001; Mullins, Rummel \& Spada, 2011). Collaboration is understood to be a coordinated activity that is the result of a continued attempt to construct and maintain a shared conception of a problem (Roschelle \& Teasley, 1995). In contrast, cooperation means that the cooperators split the task into parts, and each cooperator works with different parts.

The didactical design in this study is built on these ideas. The students collaborated by working in pairs while sharing one computer using GeoGebra. The task was designed to constitute a challenge to the students. Finally, the students were given the responsibility to create their own solution methods. The author was present to answer technical questions but did not guide the students on how to solve the task. The task was presented to the students as follows:

- Create a linear function on the formula of $y=m x+c$

- Create another linear function in a way that the corresponding graphs are perpendicular

- Formulate a rule for when two linear functions have perpendicular corresponding graphs

- Test your rule on different functions with different slopes. Explain why it works

The task was pilot-tested and found suitable for 16- to 17-year-old students. 


\section{Participants and Data Collection}

Sixteen students from the science program at a Swedish upper-secondary school volunteered. They were 16-17 years old - eight girls and eight boys. They had earlier experiences of linear functions from lower-secondary school, but they had no recent teaching on the issue. They were informed according to the ethical directives of the Swedish Research Council (2001).

The students solved the task in pairs in a room beside the regular classroom. They had no experience of working with GeoGebra and used a prepared GeoGebra file where all tools were disabled except for the pointer, the "layer-mover" and the angletool, since experiences from earlier studies (Granberg \& Olsson, 2015) and pilot-tests have indicated that some students become disoriented in the solving process while exploring different tools. They had a short introduction to GeoGebra as well as to how to submit formulas, how to change an algebraic expression and how to use the visible tools. In situations in which students became stuck, the author encouraged them to explain their ideas and strategies to help them move on in their work. Finally, when the students had solved the task (or had given up), they were invited to explain why they thought that their strategies had or had not been successful. The data were screen recordings, with integrated voice and video recording.

\section{Analysis Method}

Research Question 1 concerns the contribution of the students' reasoning for utilizing the feedback generated by GeoGebra. Students' reasoning was categorized using Lithner's (2008) framework of creative and imitative reasoning. How students used the feedback from GeoGebra was examined using the concepts of verificative and elaborative feedback (Shute, 2008). Thereafter, the relationships between students' verificative and elaborative use of feedback and their engagement in creative and imitative reasoning were examined. Research Question 2 concerns how the results from RQ1 relate to students' problem-solving success. This was analysed by considering whether important decisions in the solving process were consequences of certain reasoning and use of feedback from GeoGebra. The analysis methods indicated here will be elaborated in the following text.

Structuring Data. The data, consisting of dialogue, computer interactions and gestures, were transcribed into written text. To discuss students' reasoning and their use of feedback from GeoGebra in the context of their problem-solving success, the eight pairs were divided into two groups: those who reached a reasonable solution, that is to say those who constructed a rule, and those who did not. As the next step, the transcripts were divided into episodes according to Schoenfeld's six phases of problem-solving: that is to say, reading, analysing, planning, implementing, exploring and verifying. To map the students' path through their problem-solving, possible decision points were identified - that is to say, junctions between episodes, occasions on which new information arose from computer activities or students' discussions, and sequences accompanied by difficulties. Actual decisions, when what students said or did indicated how to proceed, were noted. These parts were used to consider how the decisions contributed to solving parts of the task and if information gained from solving parts of 
the task was used to answer the main question of the task. Thereafter, the students' reasoning in these phases and transitions was analysed.

Students' Reasoning. Lithner's (2008) framework was used to classify the students' reasoning into IR or CMR. The students' dialogues, interactions with GeoGebra and gestures in each phase were examined, and units of argumentation were identified. The characteristics of the argumentation - that is to say, the implicit or explicit justifications of the strategy choices and the strategy implementations-were used to determine whether the reasoning met with the understanding of what is characterized as imitative or creative reasoning. The students' reasoning was considered CMR if there were signs of creating (for the students) a new solution method (that may contain some elements of IR, though not only) and if their argumentation was anchored in intrinsic mathematical properties. The reasoning was categorized as imitative reasoning if the (sub-) task solutions were based essentially on familiar facts and/or procedures only.

Students' Use of Feedback. Finally, how students used the feedback from GeoGebra was examined using the concepts of verificative and elaborative use of feedback (Shute, 2008). Dialogues and gestures during phases before and after each computer activity were noted. A computer activity in this study includes the students' input and the outcomes displayed by GeoGebra. Before this point, the students plan (planning phase) what to submit to the program, and afterwards, the students may interpret the outcome and discuss how to proceed (verificative and analytic phase). What they said in a planning phase when they predicted the outcome of a computer activity was interpreted as being preparation for using the information from GeoGebra as verifying feedback. After a computer activity, in the verificative phase, students could use the feedback from GeoGebra verifiably, which was identified as what they said in terms of success or failure in reaching the expected (sub-) goal. If they used the verification information to explain, extend pre-knowledge, plan for how to proceed with the task solving, etc., then they were considered to be using the information from the program elaborately and to be entering the analytic phase. Finally, the situations of preparing activities and using feedback from GeoGebra were considered in the context of whether the reasoning was characterized as being CMR or IR.

To answer RQ1, the use of feedback (verifiably and/or elaborately) was associated with the characteristics of IR or CMR during the planning of the activity and with reasoning when using feedback.

To answer RQ2, the reasons for students' success or failure in solving the task were related to decisions in the solving process that the students made or could have made. Whether the success or failure was related to the characteristics of reasoning and use of feedback was then considered.

\section{Analysis}

All eight pairs were engaged in the problem-solving process; however, not all of them solved the task. Four pairs found a reasonable solution for the main task. They used possible decision points to solve sub-problems and used new information to solve the 
following sub-problems and the main task. Two pairs did not find a reasonable solution for the main task. They solved some sub-problems but made less use of their experiences from solving these sub-tasks. The remaining two pairs started in the same manner as the less successful pairs but changed strategies and completed the task in the same way as the more successful pairs. In the following discussion, sequences from one pair of each category will be analysed with respect to their reasoning and utilization of feedback. Because none of the chosen pairs had a clear understanding of the formula $y=m x+c$, they all needed to clarify the properties of the formula. The following examples are from such sequences.

\section{Alma and Ester}

Alma and Ester had an exploratory approach and they successfully solved the task. Before the first algebraic submission into GeoGebra, they had a discussion as to where they wanted the first graph to be situated. After two attempts to create a perpendicular graph to $y=7 x-1$, they came to the conclusion that they needed to understand the formula $y=\mathrm{m} x+\mathrm{c}$. By manipulating existing formulas and observing the graph, they found out that $\mathrm{m}$ affects the slope of the graph and $\mathrm{c}$ affects its intersection with the $y$ axis. When that was sorted out, they used these findings to create a number of perpendicular graphs. Finally, they observed that the product of the $x$-coefficients in all their examples were -1 and proposed $m_{1} \times m_{2}=-1$ as the rule.

Episodes and Decision Points. During their task solving, Alma and Ester went through episodes of reading, exploring, planning/implementing, analysing and verifying. They had possible decision points at the junctions of episodes and when the computer activity generated new information. Two of those decision points in particularly helped them with their problem-solving. The first of these decision points emerged when they realized that they did not fully understand the formula $y=m x+$ c, and they decided to analyse the properties of the formula. The second decision point arose when they had difficulties in finding a function with perpendicular graphic representations to the graph of $y=7 x-1$, and they decided to change the function to $y=2 x-1$ because 2 is easier to divide than 7 . It was also clear that they used information from these episodes of analysis later in the task-solving process. In the next paragraph, their first episodes of exploring will be analysed.

Reasoning. After reading the instructions, they initiated an exploring episode as follows:

1. Ester: Well let's just submit something...

2. Alma: $y$ is equal to seven...

3. Ester: That means it's going to be very much like this (a moving gesture almost vertically, in front of the screen)

Their suggestion to choose seven as the $x$-coefficient is followed by a prediction of the graphical appearance on the screen. They created the strategy themselves and what Ester says along with her gesture can be interpreted as being predictive argumentation. This strategy of suggesting something followed by a prediction of the result supported 
by argumentation reappeared several times during their work. Some predictions were followed up by verificative argumentation: for example, " $m=7$ means the line must increase by 7 every step to the right" or "this one must have m less than 1 because you go more steps horizontally than vertically". The reasoning of these students is classified as CMR since their reasoning is novel (i.e. is not a familiar algorithm) and is based on predictive argumentation as to where the graph would be situated and verificative argumentation as to why the graph appeared as it did.

Feedback. The following excerpt, considered an episode of analysis, exemplifies how Alma and Ester used the information after submitting the function $y=-3 x-1$, which they predicted to have "negative but less slope than $y=7 x-1$ ":

1. Alma: This is not $90^{\circ} \ldots$

2. Ester: No, it's not... but let's measure it to see how far off we are [uses GeoGebra's angle tool to measure the angle]...

After a discussion resulted in the conclusion that the constant term does not affect the slope of the function and that the slope depends only on $\mathrm{m}$, the $x$-coefficient:

\section{Alma: We must concentrate on m....}

After an analysis of different examples of submitted functions, Alma summarized using $y=2 x-1$ and $y=7 x-1$ as references:

4. Alma: Well, if we start at minus one.... This one has $m=2 \ldots$. Then, you go one step to the right and then two upwards [counting squares with the mouse].... And this has $\mathrm{m}=7 \ldots$ if you go one step to the right, you go seven upwards [counting squares with the mouse]....

First, they used feedback from GeoGebra for verification, concluding that they did not have a perpendicular line. They then initiated an attempt to elaborate on the result. This led them to an episode of analysis in which they elaborated on the feedback and investigated how $\mathrm{m}$ and $\mathrm{c}$ affect the graphical representation. During their work, these students frequently discussed and elaborated on the received feedback, and based on this, they adjusted their strategies. This indicates that they were using the feedback from the software both as verificative and elaborative feedback.

\section{Relationships Between Reasoning, Feedback and Success in Problem-} Solving. Alma and Ester frequently used CMR to predict the outcome of the computer activities, and they used the feedback from GeoGebra for both verification and elaboration. Furthermore, these students always related their elaborations to their predictions. This indicates a relationship between CMR and elaboration on feedback from GeoGebra. It seems that predictions of computer activities that are founded in CMR provide a basis for using the received feedback elaborately.

Alma's and Ester's decisions to examine the formula $y=m x+c$ and to replace the $x$ coefficient of 7 with 2 are considered important for solving the task. Both decisions were made in episodes of analysis and were preceded by elaboration on feedback based 
on CMR. Information from analysis was then used to answer the main question of the task. The use of CMR by these students and their elaborative use of feedback in the episodes of analysis seem important for their success in solving the task.

\section{Bertil and Isak}

Bertil and Isak had an exploratory approach. They solved some subtasks, but they did not solve the main task. Their efforts to find a path to a solution initially meant that they tried several different linear equations. Their first example of a perpendicular graph corresponding to the $x$-coefficients 1 and -1 was found rather quickly. They did not try to understand why the example resulted in perpendicular graphs. Instead they continued submitting different linear equations, none of them resulting in perpendicular graphs. They had one more articulated approach; they tried to predict where the intersections to both the $y$-axis and the $x$-axis would be from the values of the $x$-coefficient and the constant term. When it was not verified by the information from GeoGebra, they abandoned the idea without trying to understand why it did not work. After 50 min, they gave up.

Episodes and Decisions. During their task solving, Bertil and Isak went through episodes of reading, exploring and planning/implementing. Possible decision points were junctions of episodes and when the computer activity generated new information. Their first decision was to submit $y=6 x-3$, followed by them saying that the function ought to have less slope. After some manipulation, they agreed on and submitted $y=x$ -3 . Then, they submitted $y=-x-3$, which they stated was perpendicular to $y=x-3$. The decision to change $y=6 x-3$ to $y=x-3$ made the sub-task easier. This decision allowed them to create $y=-x-3$ rather easily, only changing the m-value from positive to negative. The decision allowed them to find a solution to the sub-problem of creating two perpendicular lines. However, no trace was found of using gained knowledge to solve other sub-problems or to answer the main question.

Reasoning. After reading the task instruction, Bertil and Isak went on to implement an example of a linear function. The following excerpt is the beginning of their conversation after the first implementing episode:

1. Bertil: If we have.... sort of.... y equal to.... six....

2. Isak: [types $y=6] \ldots . \ldots$... isn't it.... plus....

3. Bertil: Minus.... because we want to have it down here [points with the mouse cursor at $(0,-3)] \ldots$...

4. Isak: Ok.... [completes $y=6 x-3$ and pushes the enter button].... like this.... sort of...

5. Bertil: Then we must have one going in this direction [pointing with the mouse cursor negative diagonally on the screen]...

The strategy of submitting a function to have a reference from which to proceed was created by them. Line 3 predicts the intersection to the $y$-axis, but there is no articulated argumentation as to how the submitted function will contribute to the solution. As soon as the enter button is pushed, they begin looking for a perpendicular line without 
discussing the result of the computer activity $(y=6 x-3)$. This approach is characteristic of their reasoning throughout the entire procedure of solving the task. Although they occasionally create solution strategies and occasionally predict outcomes of computer activities, the lack of argumentation and superficial basis (or lack thereof) in mathematics means that their reasoning cannot be classified as CMR. It is not clear what the purpose of choosing the function $y=6 x-3$ was. Pointing at $(0,-3)$ seems to predict an intersection with the $y$-axis (line 3 ), which may build on that students remember that $\mathrm{c}$ determines the intersection point with the $y$-axis, but the reason behind the choice of 6 as $x$-coefficient is not clear from the data. Strategies for recalling memorized facts and procedures imply less of a necessity for argumentation, which is characteristic of IR.

Feedback. In the example above on line 3, there is a prediction as to the intersection with the $y$-axis, which is consistent with the result of the activity. However, they do not comment on this result - that is to say that the graph intersected in actual fact at $(0,-3)$. This approach is an example of using feedback verifiably. The following excerpt is an example from the same episode. The intersection with the $x$-axis $(0.5,0)$ for the function $y=6 x-3$ is not what they expected:

1. Bertil: Wait... there it is minus three [points at $(0,-3)]$... why is this situated here, then [points at $(0.5,0)] \ldots$ ?

2. Isak: Should we... should we have ten instead... ?

3. Bertil: Yes, ... type that....

4. Isak: Yes [submits $y=10 x-3] \ldots$ this is even steeper... but let's have.... one.... [submits $y=1 x-3] \ldots$

It seems that the intersection with the $y$-axis is what they expected, but they question the intersection with the $x$-axis at $(0.5,0)$. Instead of trying to understand why the intersection is at $(0.5,0)$, they repeatedly change the $x$-coefficient (line 4$)$ until they have the $45^{\circ}$ graph associated with $y=x-3$. There are no attempts to explain why an $x$ coefficient yields a certain slope. This is considered to be using feedback only verifiably, not elaborately. Using feedback only verifiably and replacing functions without discussion is characteristic of this entire task-solving session.

Relationships of Reasoning, Feedback and Success in Problem-Solving. The relationship between reasoning and feedback is illustrated by Bertil and Isak, who had no argumentation in their preparations of computer activities and who used feedback solely verifiably. The lack of argumentation disqualifies the reasoning as CMR. A consequence of the lack of predictive argumentation is that they have no clear perception of what feedback they can expect, which makes it difficult for them to elaborate on the feedback when it appears on the screen. This in turn is one reason for their failure to solve the task.

\section{Olga and Leila}

Olga and Leila's initial strategy could be described as imitative - trying to remember facts and procedures. In the first half of the task-solving process, they solved some sub-problems, 
but they did not reach an answer to the main question. After $40 \mathrm{~min}$, they changed strategy. They started to create solution methods and to analyse the received feedback. Eventually, they presented $m_{2}=-1 / m_{1}$ as an answer to the main question, which was elaborated into $m_{1}$ $\times \mathrm{m}_{2}=-1$. The following analysis is separated into two parts, before and after the strategy change. The second half will be described as a summary, focusing on the main causes for their success in solving the task.

Episodes and Decisions in the First Half. In the first half of the task-solving session, Olga and Leila went through episodes of reading, exploring and planning/implementing. Possible decision points were junctions between episodes, when the computer activity generated new information and sequences with difficulties. Their first decision was to implement $y=2 x-2$, the graph of which was supposed to intersect the $y$-axis at -2 and the $x$-axis at 2 . They did not try to analyse why it did not appear as they expected. Instead, they attempted to create a perpendicular line by submitting $y=-x-1$, which led to a decision to change $y=2 x-2$ into $y=x-1$. The decisions made them solve the part of the task that involved creating two perpendicular lines. However, no trace was found of their gained knowledge to solve other sub-problems or to answer the main question.

Reasoning in the First Half. The excerpt is from their first conversation after reading the instructions. It is considered an exploration of the conditions for the task:

1. Olga: c was where it intersected the $y$-axis...

2. Leila: Yes...

3. Olga: Yes, it was... But, what is $\mathrm{m} . .$. ?

4. Leila: $m$ was that the value in between...?

5. Olga: Yes... the difference when you go...

6. Leila: Yes...

7. Olga: Eh... What should I write then...?

What they say on lines 1 and 3 and their attempts to explain on lines 4 and 5 indicate that these students are trying to remember how c affects the intersection with the $y$-axis and how to calculate the $x$-coefficient. The articulated facts are not coherent, and there is no argumentation for why these facts may help to solve the task. This is characteristic of imitative reasoning. Only the utterance that "c was where it intersected the $y$-axis" is used in their first implementation, as exemplified in the next excerpt:

8. Leila: Should we make it easy and take $y=-2$ and $x=2$ [pointing with the mouse at $(0,2)$ and $(2,0)] \ldots$ ?

9. Olga: Yes, ... go ahead...

10. Leila: [writes $y=2 x+2$ ] ... No... minus [change and submit $y=2 x-2$ ] . hmm... 11. Olga: Yes... and a graph perpendicular to this must go ...

Line 8 indicates a prediction that the graph would intersect the $y$-axis at -2 and the $x$-axis at 2 . Their argumentation does not have a basis in mathematics. Their idea is merely to make the implementation easier. There is no argumentation for why the graph did not appear as expected. A few lines down, similar behaviour is observed: 
12. Olga: No.... that is not perpendicular.... It is too large.... But, write $y=-x-1 \ldots$. 13. Leila: [submits $y=-x-1$ ] this is not $90^{\circ} \ldots$

14. Olga: No... but we can change $y=2 x-2$ into $y=x-1 \ldots$

Instead of analysing why the graphs did not intersect perpendicularly, they changed their first function $y=2 x-2$ into $y=x-1$. This seems to be a decision based on intuition; there is no argument for why it solved the sub-task. The approach of trying to remember how the constant term and $x$-coefficients affect the graph and the lack of predictive and verificative argumentation classify the reasoning as IR.

Feedback in the First Half. The first computer activity on line $10, y=2 x-2$, did not result in the intersection with the $x$-axis that they predicted. Feedback was not explicitly used verifiably or elaborately. It may have been used implicitly as a reference to plan for a perpendicular line. Feedback from the next activity (line 12), $y=-x-1$, was used verificatively, stating that the graph was not perpendicular to $y=2 x-2$. They changed $y=2 x-2$ to $y=x-1$ (line 14) without presenting any arguments as to why. It seems that the visual feedback made them guess $y=x-1$ should be perpendicular to $y=-x-$ 1. The use of feedback, to suggest $y=x-1$ is perpendicular to $y=-x-1$, is not considered elaborative when there is no articulated attempt to understand why the lines were initially not perpendicular. This example of using feedback merely verifiably is characteristic of the first half of Olga and Leila's task solving.

\section{Relationships of Reasoning, Feedback and Success in Problem-Solving in the First}

Half. The few predictions they articulate (for example, that the c-value indicates intersection with the $y$-axis and, wrongly, that the $x$-coefficient indicates the intersection with the $x$-axis) are not supported by predictive argumentation, and the feedback from GeoGebra (for example, the graph associated with $y=2 x-2$ ) is not elaborated on. It seems that the lack of articulated predictive argumentation may have caused difficulties for the students in elaborating on feedback and using verificative argumentation.

The reason behind Olga and Leila's failure to solve the task in the first half of the session is that they did not try to understand why the feedback from GeoGebra did not verify their predictions. There is some argumentation, but it is superficial and does not have a basis in mathematics (for example, the choice of $y=2 x-2$ because it would "make it easy"). The lack of predictive argumentation also means that they do not have a basis for analysis of unexpected results of computer activities.

Second Half Change in Approach. In the first half, Olga and Leila did not manage to create perpendicular lines with $x$-coefficients other than 1 and -1 . The episodes were labelled as either implementing or exploring. They increasingly used their own solution methods, but there was no or only superficially anchored argumentation and no elaborative use of feedback. The turning point occurred in the second half, after about $40 \mathrm{~min}$. They managed by trial and error to create perpendicular lines by submitting the functions $y=2 x-4$ and $y=-0.5 x-4$. They hypothesized that one $x$-coefficient must be a fourth of the other "but negative" to create perpendicular lines. They tried this on several examples with other $x$-coefficients without success for approximately $10 \mathrm{~min}$. This is what Schoenfeld (1985) describes as a possible decision point based on information indicating 
that something is wrong. For the first time, Olga and Leila performed what can be considered as an analysis:

1. Olga: I think we started out the wrong way round.... We are looking for a pattern that does not exist.... this one affects the slope [pointing at the $x$-coefficient]... and this one the intersection with the y-axis [pointing at the constant term]

2. Leila: And $m$ affects the angle...

3. Olga: But why are these angles equal [pointing at the examples on the screen]?

4. Leila: But we said that c doesn't matter, we can move them here... and there... (pointing with her finger at different areas on the screen)

5. Olga: So it is the slope that matters... and the relation between two different slopes...

The sequence above is crucial for solving the task because they initiated an analysis of how $\mathrm{m}$ and $\mathrm{c}$ affect the graphical representation of the function (line 1), and they decided to focus on the relationship between the two $x$-coefficients of two perpendicular functions (line 5). The next excerpt exemplifies their changed approach to reasoning:

1. Olga: What do the two examples have in common $(y=x-1$ and $y=-x-1, y=2 x$ -4 and $y=-0.5 x-4) \ldots$ ?

2. Leila: They are like opposites...

3. Olga: One divided in two is zero point five....

4. Olga: Yes... and one divided in one is one... but minus...

5. Leila: That's it... one divided in one but minus...

6. Olga: Then something times something must be one... but minus.... say a number...

7. Leila: Six...

8. Olga: Then the other one must be.... one divided by six.... but minus [submit $y=$ $6 x$ and $y=-1 / 6 x] \ldots$

9. Both: Yeah...

Their strategy to find the relationship between the $x$-coefficient of two perpendicular functions generated a hypothesis (line 6). To examine their idea, they created a computer activity (line 8) using predictive argumentation based in mathematics. The reasoning in this sequence is classified as CMR. The next excerpt exemplifies that their creative and predictive reasoning before the computer activity prepared them to elaborate on feedback:

10. Olga: Alright... what do we have... six and a sixth...

11. Leila: And one of them is minus...

12. Olga: Then the m's times each other must be minus one...

13. Leila: Let's try $m$ is equal to five...

On lines 10-11, they used feedback verifiably, stating that their prediction was correct. On line 12, they elaborated on feedback based on their predicative argumentation (lines 4-6) and suggested an answer to the main question of the task. On line 13, they initiated an activity to verify their idea and, therefore, the answer to the main 
question. After this excerpt, they verified their idea using several examples and concluded that the task was solved.

Relationships Among Reasoning, Feedback and Success in Problem-Solving After

Changing Approach. The relationship between reasoning and feedback in the second half of the session is that the planning of computer activities includes the creation of strategies supported by predictive argumentation anchored in mathematics - that is to say, CMR. These strategies are then implemented, and the feedback generated by the computer activities is elaborated in the sense that students use CMR to explain why the feedback does or does not verify predictions. The example above shows that Olga and Leila's predictive argumentation is the basis for the elaboration on feedback. This indicates that the argumentation behind the prediction prepared them for using the feedback: that is to say, the activities $(y=6 x$ and $y=-1 / 6 x$ ) verify the prediction of creating perpendicular lines.

The reason behind Olga and Leila's success in solving the task is that, after a long period of fruitless trials, they performed an analysis of their examples, $y=x-1$ and $y=$ $-x-1, y=2 x-4$ and $y=-0.5 x-4$. This analysis initiated a change in reasoning to CMR: that is to say, they started to give arguments for their strategies and predictions. When the analysis turned into implementation, they started to elaborate on feedback; for example, they discussed how to choose $x$-coefficients to provide perpendicular lines. There is a clear distinction between their reasoning before and after the sequence in which they tried several examples with different $x$-coefficients on the assumption that one $x$-coefficient should be the negative fourth of the other. As long as they did not support this prediction by argumentation, they did not come closer to a solution. After analysis of the examples, they justified the relationship that one $x$-coefficient must be minus one divided by the other. Through elaboration on feedback, they continued towards a solution to the task; the product of the $x$-coefficients must be -1 .

\section{Conclusions}

The conclusions are presented in line with the research questions addressing interpretation of feedback, predictive and verificative argumentation, and success in solving the task.

\section{Contributions of Students' Reasoning to the Utilization of Feedback from}

GeoGebra. The results from the study show that differences in utilizing feedback generated by activities in GeoGebra can be referred to different characteristics of reasoning. For example, Alma and Ester, and Bertil and Isak all started by submitting similar formulas $(y=6 x-3$ and $y=7 x-1)$ and consequently received similar feedback from GeoGebra, but while Bertil and Isak immediately and without reflection erased the function and submitted another one, Alma and Ester elaborated and uncovered implicit meanings of the feedback by examining the features of the $x$-coefficient and constant term. The differences between the two pairs were that Alma and Ester's reasoning frequently included predictions of the outcome from activities and justifications supporting elaboration on feedback, while the reasoning of Bertil and Isak essentially did not include any of these components.

Upon a further look into the characteristics of the solutions, it becomes apparent that students who elaborate on feedback and present verificative argumentation supporting claims in solutions are those who explicitly predict outcomes from computer activities 
before submitting input to GeoGebra. Olga and Leila exemplify both an approach without predictive argumentation (first half of their solution) and (in the second half) a solving strategy where every action was preceded by predictions of the outcome. Contrary to the first half, the second half included elaboration of feedback partially supported by predictive argumentation. Bertil and Isak, on the other hand, even though they seemed to have expectations as to the outcomes, never articulated any predictions or verificative argumentation.

Students' Path of Reasoning and Utilization of Feedback from GeoGebra Related to Success in Problem-Solving. In this study, the reoccurring fact is that students who solve the tasks are those who manage to elaborate on the feedback from GeoGebra. None of the students knew the relation between two linear functions with perpendicular corresponding graphs. That meant they had to formulate activities that would result in information that could be used to support the construction of a rule. However, this information from GeoGebra is often not in the form of direct answers to the questions that the students have in mind but must be interpreted, elaborated and transformed. What the successful students in this study all have in common is that their reasoning is essentially characterized as being CMR: that is to say, their reasoning includes predictive and verificative argumentation, something that was characteristic for those who utilized feedback elaborately. Both Alma's and Ester's and Olga's and Leila's (second half) path of reasoning can be described as follows: formulating input including prediction of the outcome, submitting into GeoGebra, and interpreting and elaborating on the outcome based on the prediction.

\section{Discussion and Conclusions}

The results of this study clearly indicate that interpretation of feedback from GeoGebra is crucial for success in solving the task and that prediction as to the outcome of activities in GeoGebra is important for utilizing feedback from the software efficiently. This may be explained by what Sedig and Sumner (2006) call discrete interaction with software: that is to say, there are parts in the transformation processed by software that are not transparent. For example, if the student submits a linear equation into the software, GeoGebra will create the corresponding graph but not point out the particular relationships of interest between the equation and the graph. To notice these relationships, the student needs to interpret the provided feedback generated by her action. Interacting with software through commands means the user has to formulate an input object (Holst, 1996), and formulating input may be more or less associated with a clear and specific purpose to receive useful information from the activity. As seen in this study, articulated predictions and hypotheses of the outcome provide the basis both for submitting suitable input and for interpreting feedback from GeoGebra efficiently.

\section{How Reasoning Contributes to the Utilization of Feedback}

The study shows that GeoGebra has the potential to help in the solving of non-routine tasks through quickly and exactly transforming and displaying representations of 
mathematical objects simultaneously. For example, a submitted algebraic linear function is transformed into a graph, and both the algebraic and graphical representations will be displayed side by side. Utilizing the potential for task solving means creating mathematical objects that support the solution. Since the management of procedures is taken care of by the software (in the case of this study, the transformation of a linear equation into its graphical representations), GeoGebra is a tool to help students express, adjust and refine their thinking (see Hoyles et al., 2004). Falcade et al. (2007) point out that working with dynamic software can be used not only to explore mathematical objects but also to support reasoning leading to the solving of mathematical problems. From the point of view of reasoning, successful interaction with GeoGebra resonates well with the concept of CMR (constructions of new solutions supported by arguments). That is, the student needs to create the solution method, and the more thorough the input is prepared (including predictions, hypotheses and predictive argumentations), the better prepared she is to interpret and utilize the feedback generated (including conclusions supported by verificative argumentations).

\section{Implications for Mathematics Education}

In this study what is recurrent is that students who solve the task are those who manage to elaborate on the feedback from GeoGebra. While there were no signs that any of the students knew the rule in advance or knew how to create perpendicular graphs, they all had to (1) figure out how to create at least two examples of linear functions with corresponding perpendicular graphs, (2) draw conclusions in terms of the relationship between $x$-coefficients when two functions have perpendicular graphical representations, and (3) formulate a rule. That means that students have to organize and extend their current knowledge about linear function and its representations. In Swedish schools, the relationship between linear functions that have corresponding perpendicular graphs is usually taught in years 10-11 as part of the science and technology programs. The most common way is for the teacher and/or textbook to present and justify the rule $m_{1} \mathrm{x} \mathrm{m}_{2}=-1$ followed by a couple of tasks where the rule is applied. Some textbooks present exploratory tasks including stepwise instructions that, if followed correctly, will create (either by pen and paper or some technological means) representations of linear functions usable as references to formulate the rule. This study shows that students themselves, with the support of GeoGebra, are capable of creating the necessary representations and of drawing conclusions to the rule without stepwise instructions. On the other hand, there were students who failed to solve the task. In regular teaching situations, these students would probably have interacted with the teacher, and they would probably have had textbooks to help them. If the teacher and/or textbook provides the students with a solution method, they would probably solve the task successfully, but most likely their reasoning would turn into being imitative, that is, they would have adapted and applied the solution method without justification based in mathematics. If a purpose is to engage students in CMR, the support to students who meet obstacles must be carefully prepared. According to the results of this study, students should be encouraged to predict the outcome of activities in GeoGebra. Furthermore, instead of explaining how to solve the task, the teacher should ask the students to justify their solution methods and solutions. 
Open Access This article is distributed under the terms of the Creative Commons Attribution 4.0 International License (http://creativecommons.org/licenses/by/4.0/), which permits unrestricted use, distribution, and reproduction in any medium, provided you give appropriate credit to the original author(s) and the source, provide a link to the Creative Commons license, and indicate if changes were made.

\section{References}

Brousseau, G. (1997). Theory of didactical situations in mathematics. Dordrecht, The Netherlands: Kluwer Academic Publishers.

Falcade, R., Laborde, C. \& Mariotti, M. A. (2007). Approaching functions: Cabri tools as instruments of semiotic mediation. Educational Studies in Mathematics, 66, 317-333.

Granber, C. \& Olsson, J. (2015). ICT-supported problem solving and collaborative creative reasoning: Exploring linear functions using dynamic mathematics software. The Journal of Mathematical Behavior, 37, 48-62.

Healy, L. \& Hoyles, C. (2001). Software tools for geometrical problem solving: Potentials and pitfalls. International Journal of Computers for Mathematical Learning, 6(3), 235-256.

Hohenwarter, M., \& Fuchs, K. (2004). Combination of dynamic geometry, algebra and calculus in the software system GeoGebra. Retrieved from http://www.geogebra.org/publications/pecs_2004.pdf [5.6.16].

Hollebrands, K. F. (2007). The role of dynamic software programs for gepometry in the strategies high school mathematics students employ. Journal of Research in Mathematics Education, 34(2), 164-192.

Holst, S. J. (1996). Directing learner attention with manipulation styles. In M. J. Tauber (Ed.), Proceedings of the CHI '96 Conference Companion on Human Factors in Computing Systems: Common Ground (CHI '96) (pp. 43-44). Vancouver, Canada: ACM Press.

Hoyles, C., Noss, R. \& Kent, P. (2004). On the integration of digital technologies into mathematics classrooms. International Journal of Computers for Mathematical Learning, 9(3), 309-326.

Jones, K. (2000). Providing a foundation for deductive reasoning: Students' interpretations when using Dynamic Geometry software and their evolving mathematical explanations. Educational Studies in Mathematics, 44(1), 55-85.

Lesh, R. \& Yoon, C. (2004). Evolving communities of mind-in which development involves several interacting and simultaneously developing strands. Mathematical Thinking and Learning, 6(2), 205-226.

Lithner, J. (2008). A research framework for creative and imitative reasoning. Educational Studies in Mathematics, 67(3), 255-276.

Lou, Y., Abrami, P. C. \& d'Apollonia, S. (2001). Small group and individual learning with technology: A meta-analysis. Review of Educational Research, 71(3), 449-521.

Mariotti, M. A. (2000). Introduction to proof: The mediation of a dynamic software environment. Educational Studies in Mathematics, 44(1\&2), 25-53.

Moreno-Armella, L. M., Hegedus, S. J. \& Kaput, J. J. (2008). From static to dynamic mathematics: Historical and representational perspectives. Educational Studies in Mathematics, 68(2), 99-111.

Mullins, D., Rummel, N. \& Spada, H. (2011). Are two heads always better than one? Differential effects of collaboration on students' computer-supported learning in mathematics. International Journal of Computer-Supported Collaborative Learning, 6(3), 421-443.

Pólya, G. (1954). How to solve it: A new aspect of mathematical method. Princeton, NJ: Princeton University Press.

Roschelle, J. \& Teasley, S. D. (1995). The construction of shared knowledge in collaborative problem solving. In C. O’Malley (Ed.), Computer supported collaborative learning (pp. 69-97). New York, NY: Springer.

Sacristán, A. I., Calder, N., Rojano, T., Santos-Trigo, M., Friedlander, A., Meissner, H. \& Perrusquía, E. (2010). The influence and shaping of digital technologies on the learning and learning trajectories of mathematical concepts. In C. Hoyles \& J. B. Lagrange (Eds.), Mathematics education and technologyRethinking the terrain (pp. 179-226). New York, NY: Springer.

Sangwin, C., Cazes, C., Lee, A., Wong, K. L. \& Friedlander, A. (2010). Micro-level automatic assessment supported by digital technologies. In C. Hoyles \& J. B. Lagrange (Eds.), Mathematics education and technology-Rethinking the terrain (pp. 179-226). New York, NY: Springer.

Schoenfeld, A. (1985). Mathematical problem solving. Orlando, FL: Academic.

Sedig, K. \& Sumner, M. (2006). Characterizing interaction with visual mathematical representations. International Journal of Computers for Mathematical Learning, 11(1), 1-55.

Shute, V. J. (2008). Focus on formative feedback. Review of Educational Research, 78(1), 153-189. 
Stahl, G. (2002). Contributions to a theoretical framework for CSCL. In G. Stahl (Ed.), Proceedings of CSCL 2002 (pp. 62-71). Boulder, CO: Boulder.

Swedish Research Council. (2001). Ethical principles of research in humanistic and social science. Retrieved from http://vr.se [10.10.12].

Villarreal, M. E. \& Borba, M. C. (2010). Cellectives of humans-with-media in mathematics education: Notebooks, blackboards, calculatots, computers, and ... notebooks throughout 100 years of ICMI. ZDM Mathematics Education, 42(1), 49-62. 